\title{
Tecnura
}

\section{Selección de tecnologías para el tratamiento de aguas residuales municipales}

\section{Selection of technologies for municipal wastewater treatment}

\author{
Juan Pablo Rodríguez Miranda ${ }^{1}$, César Augusto García Ubaque², Janneth Pardo Pinzón ${ }^{3}$
}

Fecha de recepción: 22 de abril de 2015

Fecha de aceptación: 24 de agosto de 2015

Como citar: Rodríguez Miranda, J. P., García Ubaque, C. A., \& Pardo Pinzón, J. (2015). Selección de tecnologías para el tratamiento de aguas residuales municipales. Revista Tecnura, 19(46), 149-164. doi:10.14483/udistrital. jour.tecnura.2015.4.a12

\section{Resumen}

La planificación ambiental hídrica en cuencas hidrográficas debe involucrar diferentes elementos para la descontaminación del cuerpo de agua receptor. Es por ello que para la selección de las plantas de tratamiento de aguas residuales municipales en países en vía de desarrollo se deben considerar, entre otros aspectos, la composición típica del agua residual cruda, la eficiencia de remoción de contaminantes por tipo de tecnología, indicadores de desempeño por tecnología, aspectos ambientales sobre localización y la estrategia espacial para la localización. Este artículo presenta la metodología desarrollada por los autores, construida con base en los aspectos técnicos, económicos y ambientales, como una herramienta para la toma de decisión de inversiones futuras de plantas de tratamiento de aguas residuales municipales con elementos multidisciplinarios.

Palabras clave: aguas residuales, tratamiento, tecnología, selección, planificación ambiental.

\begin{abstract}
In water environmental planning in watersheds should contain aspects for the decontamination of receiving water body, therefore the selection of the treatment plants municipal wastewater in developing countries, you should consider aspects of the typical composition raw wastewater pollutant removal efficiency by technology, performance indicators for technology, environmental aspects of localization and spatial localization strategy. This methodology is built on the basis of technical, economic and environmental attributes, such as a tool for decision making future investments in treatment plants municipal wastewater with multidisciplinary elements.
\end{abstract}

Keywords: selection, technology, treatment, wastewater environmental planning.

Ingeniero sanitario y ambiental, magíster en Ingeniería Ambiental, estudiante del Doctorado en Ingeniería, Facultad de Ingeniería Universidad Distrital Francisco José de Caldas. Profesor Asociado, Universidad Distrital Francisco José de Caldas. Director del grupo de investigación AQUAFORMAT. Contacto: jprodriguezm@udistrital.edu.co

Ingeniero civil, magíster en Ingeniería Ambiental, doctor en Ingeniería. Profesor Asociado, Universidad Distrital Francisco José de Caldas. Director del grupo de investigación GIICUD. Contacto: cagarciau@udistrital.edu.co

3 Ingeniero catastral y geodesia, magíster en Ciencias de la Información y las Comunicaciones. Profesor Asistente, Universidad Distrital Francisco José de Caldas. Contacto: jpardop@gmail.com. 


\section{INTRODUCCIÓN}

Las plantas de tratamiento de aguas residuales municipales (PTARM) se pueden considerar como un proceso productivo: las salidas son los contaminantes removidos de las aguas residuales crudas y la entrada es el costo de operación y mantenimiento de la planta). Estas plantas son utilizadas en la remoción de contaminantes presentes en el agua residual cruda, y deben responder a una alta variabilidad temporal del flujo o caudal de entrada, así como a la variabilidad de las concentraciones o componentes propios de estas aguas residuales. Esto requiere una interacción en los mecanismos biológicos, físicos y químicos entre los procesos unitarios, los fenómenos hidrodinámicos y la adaptabilidad del consorcio microbiano ante las condiciones ambientales cambiantes (Hong, 2003; Bdour A, 2009).

Debido a esto, se han definido elementos esenciales para el dimensionamiento y diseño de las PTARM (Gernaey, 2004), como la caracterización del agua residual cruda, la finalidad del modelo de la PTARM, el sistema hidráulico, el análisis del modelo en estado estacionario, la calibración del modelo, entre otros. Sin embargo, la mejor tecnología disponible de PTARM debe estar articulada con los conceptos ambientales, económicos y de sostenibilidad a largo plazo (Schoenberger, 2009); por ello, para seleccionar la mejor tecnología existen aspectos de evaluación que consideran: la caracterización del agua residual cruda o influente, la tecnología apropiada al ambiente local, el cumplimiento normativo de vertimiento, la evaluación de factores ambientales externos a la PTARM, la evaluación de la viabilidad económica, la evaluación de factores tecnológicos de operación y mantenimiento (Chung, 2013).

La metodología de selección tecnológica más apropiada para el tratamiento de las aguas residuales debe ayudar en la toma de decisiones en inversión de recursos económicos para descontaminar los cuerpos de aguas (Geldermann, 2004). Por ello esta debe estar basada en factores de sostenibilidad, el análisis del ciclo de vida y los costos asociados a la inversión, operación y mantenimiento. Una opción metodológica es la técnica de toma de decisiones multicriterio para evaluar diferentes alternativas tecnológicas de PTRAM, en condiciones complejas y con enfoques estructurados y lógicos de toma de decisión. Por ejemplo, se consideran criterios como el calentamiento global, la eutrofización, el costos del ciclo de vida (valor presente neto), disponibilidad de área $\left(\mathrm{m}^{2}\right.$ por persona), personal para la operación, robustez del sistema (confiabilidad, flexibilidad, aceptabilidad, durabilidad) y sostenibilidad (análisis del ciclo de vida, ACV) (Rodríguez, 2011; Garrido, 2013), dado que establece la estimación de los impactos ambientales acumulativos de todas las etapas del ciclo de vida de un producto o proceso.

Sin embargo, esto muchas veces no se tiene en cuenta en la selección de la tecnología más apropiada para las necesidades de energía y otros criterios de sostenibilidad (Kalbar, 2012). Por otra parte, también se pueden describir criterios como asequibilidad (capacidad económica de la comunidad, disponibilidad de terrenos para la instalación de la PTARM y la energía eléctrica disponible en la zona), aceptabilidad (eficiencia y rendimiento de la tecnología de tratamiento, manejo de subproductos) y capacidad de gestión (operación y mantenimiento y el nivel tecnológico o de habilidad requerido por el operador) (Arceivala, 2007).

La mayoría de las metodologías de selección de tecnologías de PTARM se han centrado en la eficiencia de remoción de contaminantes, la viabilidad de la tecnología, eficiencia económica y, los más actuales, del Data Envelopment Analysis (DEA), aspecto de análisis alternativo para evaluar de forma simultánea la eficiencia técnica y económica de las PTARM (Sala-Garrido, 2011; Molinos, 2010; Urkiaga, 2006; Hernández, 2009). También se han implementado modelos lineales, dinámicos y no lineales, así como el proceso de jerarquía analítica para optimizar el análisis de las alternativas de PTARM, el cual tiene en cuenta aspectos cualitativos y cuantitativos de decisión y reduce el 
nivel de comparación de factores (Pophali, 2011; Zeng, 2007; Bottero, 2011). Sin embargo, la tecnología apropiada para el tratamiento de las aguas residuales municipales deberá establecerse en función de los recursos económicos, el grado desarrollo económico de la zona de estudio, la calidad del agua residual cruda y tratada, la disponibilidad de terrenos y la facilidad o adaptabilidad para satisfacer requerimientos futuros de ampliación y optimización (Rodríguez, 2009).

En el caso de Colombia, no existe una metodología estandarizada o reglamentada para la selección de PTRAM; aun cuando en la legislación y normativa vigente se consideran algunos principios y criterios para la selección del tratamiento de las aguas residuales, específicamente no se establece una metodología apropiada. De acuerdo con lo anterior, el presente manuscrito tiene como objetivo proponer una metodología para la selección de la tecnología de tratamiento de aguas residuales municipales, que incluye atributos (cualidades o propiedades) significativas de forma racional y multipropósito que se ajustan a las fases de proyectos (idea, perfil y prefactibilidad) de PTARM, teniendo en cuenta atributos técnicos, económicos y ambientales, en contextos para países en vía de desarrollo.

\section{METODOLOGÍA}

\section{Tipo de investigación}

La investigación aplicada a esta metodología de selección de tecnologías de las PTARM es, según el análisis y alcance de los resultados, de tipo descriptivo (Vergel, 2010), dado que se describen características o propiedades de las tecnologías de las PTARM. Además, según el tiempo de ocurrencia del fenómeno, es de tipo prospectivo, debido a que la información se va registrando y analizado según ocurre el fenómeno de estudio, es decir, según se consolida la metodología para la selección de las PTARM. Además, la investigación también es de pronóstico (Hurtado J., 2000), dado que se anticiparán situaciones futuras para toma de decisiones, en condiciones de horizonte de proyecto, calidad de agua residual cruda y tratada, cumplimiento normativo ambiental vigente en la descarga de aguas residuales tratadas y el fortalecimiento institucional.

\section{Desarrollo de la metodología de selección de tecnologías}

A continuación se exponen los elementos necesarios para la elaboración de la metodología de selección de tecnologías para el tratamiento de aguas residuales municipales.

\section{Composición típica de las aguas residuales municipales}

La composición típica de las aguas residuales crudas es una de las variables para la selección de PTRAM (Metcalf \& Eddy, 2003; Hammer, 2012; Hernández, 1996; Villaseñor, 2001; APHA, 2009; Crites, 2000). En la tabla 1 se observa la diferenciación entre las concentraciones (fuerte, media, débil/ligera) según autor; de ello dependerá el grado de tratamiento, la tecnología a utilizar y la eficiencia o rendimiento esperado para el cumplimiento normativo.

\section{Eficiencias de remoción de contaminantes por tecnologías}

Las eficiencias de remoción de las diferentes tecnologías para el tratamiento de aguas residuales municipales (RAS, 2000; Metcalf \& Eddy, 2003; Eckenfelder, 2000; Crites, 2000; Fresenius, 1991; Mara, 1997; Lettinga, 1991; Von Sperling, 1996; Gloyna, 1971; Arceivala, 1986; Ferrer, 2008; Van Haandel, 1994; Yañez, 1995; Fair, 1968) pueden observarse en la tabla 2, en donde se puede seleccionar la tecnología de PTARM según los parámetros del agua residual cruda que se tenga y según el nivel o grado de cumplimiento normativo deseado. 


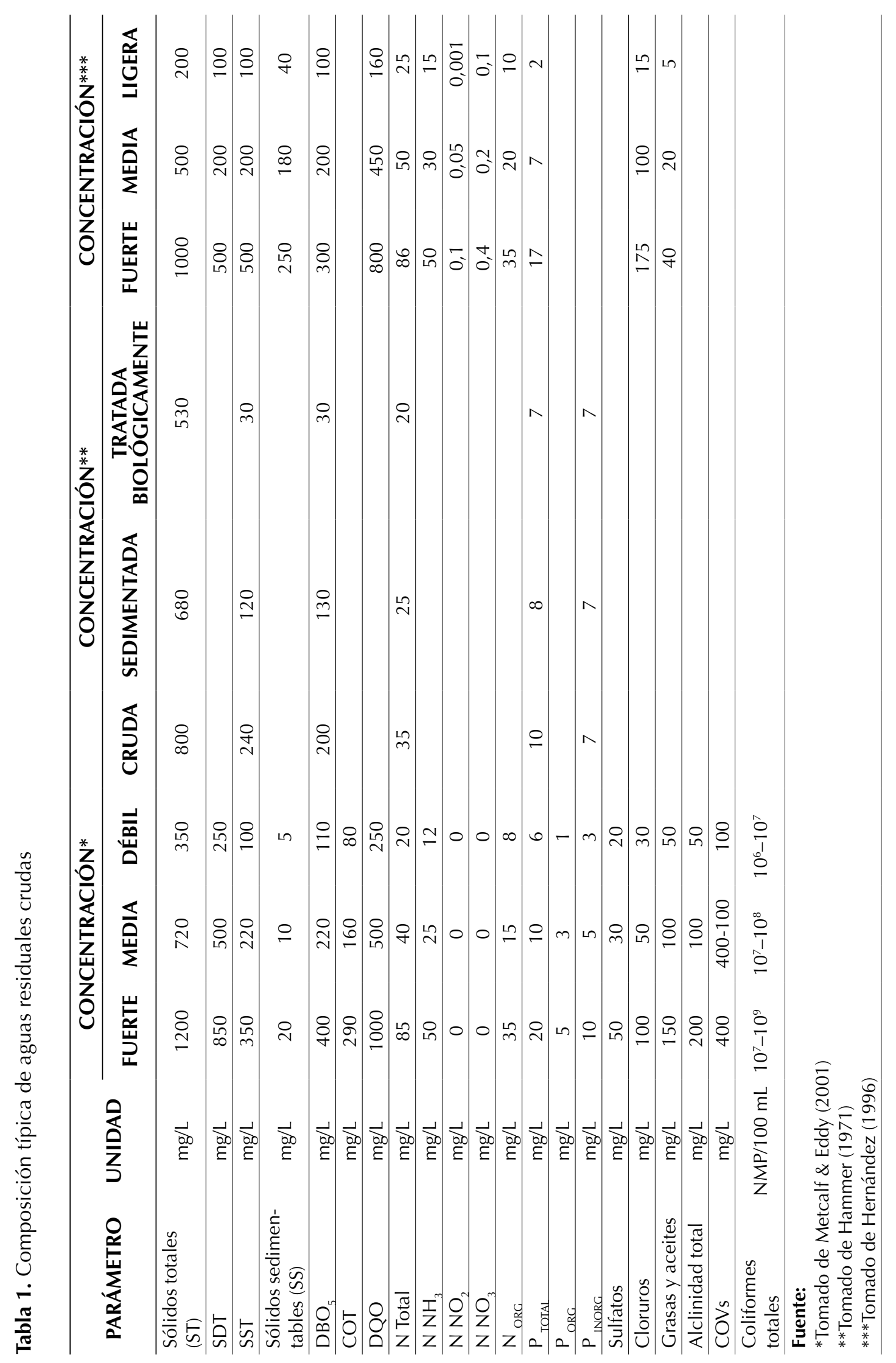


Tabla 2. Eficiencias de remoción de contaminantes por tecnologías

\begin{tabular}{|c|c|c|c|c|c|c|c|c|c|c|c|}
\hline \multirow{2}{*}{$\begin{array}{c}\text { TECNOLOGÍA } \\
\text { DE } \\
\text { TRATAMIENTO }\end{array}$} & \multirow[b]{2}{*}{ REFERENCIA } & \multicolumn{10}{|c|}{ EFICIENCIA DE REMOCIÓN (\%) } \\
\hline & & SST & $\mathrm{DBO}_{5}$ & DQO & $\mathrm{N} \mathrm{NH}_{3}$ & N ORG & $\mathrm{N} \mathrm{NO}_{3}$ & $\begin{array}{c}\mathrm{N} \\
\text { TOTAL }\end{array}$ & $\mathrm{PPO}_{4}$ & $\begin{array}{c}\mathbf{P} \\
\text { TOTAL }\end{array}$ & COLIFORMES \\
\hline \multirow[b]{2}{*}{$\begin{array}{l}\text { Desarenador } \\
\text { convencional }\end{array}$} & RAS (2000) & $0-10$ & $0-5$ & $0-5$ & & & & & & & \\
\hline & $\begin{array}{l}\text { Metcalf \& Eddy } \\
(2001)\end{array}$ & $0-10$ & $0-5$ & $0-5$ & & & & & & & \\
\hline \multirow{3}{*}{$\begin{array}{l}\text { Sedimentador } \\
\text { primario }\end{array}$} & $\begin{array}{l}\text { Metcalf \& Eddy } \\
(2001)\end{array}$ & $50-65$ & $30-40$ & $30-40$ & & $10-20$ & & & & $10-20$ & \\
\hline & Fair (1954) & $40-70$ & $25-40$ & $20-35$ & & & & & & & $25-75$ \\
\hline & Yáñez (1995 & $40-70$ & $25-40$ & & & & & & & & $25-75$ \\
\hline Tanque séptico & Batalha (1989) & $50-70$ & $40-62$ & & & & & $<10$ & & $<10$ & $<60$ \\
\hline $\begin{array}{l}\text { Tanque séptico - } \\
\text { filtro }\end{array}$ & $\begin{array}{l}\text { Von Sperling } \\
\text { (1996) }\end{array}$ & & $70-90$ & & & & & $10-25$ & & $10-20$ & $60-90$ \\
\hline Tanque Imhoff & $\begin{array}{l}\text { Tchobanoglous } \\
(2000)\end{array}$ & 50 & 40 & & & & & & & & \\
\hline \multirow{2}{*}{$\begin{array}{l}\text { Primario avan- } \\
\text { zado }\end{array}$} & Yáñez (1995) & $70-90$ & $50-85$ & & & & & & & & $40-80$ \\
\hline & Tsukamoto (2002) & $73-84$ & $46-70$ & & & & & $<30$ & & $10-20$ & $80-90$ \\
\hline \multirow[b]{2}{*}{ Filtro anaerobio } & RAS (2000) & $60-70$ & $65-80$ & $60-80$ & & & & & & $30-40$ & \\
\hline & $\begin{array}{l}\text { Rodríguez et al. } \\
\text { (2006) }\end{array}$ & & & $75-85$ & & & & & & & \\
\hline \multirow{4}{*}{ UASB } & Torres $(2000)$ & $60-80$ & $60-70$ & & & & & $10-25$ & & $10-20$ & $60-90$ \\
\hline & RAS (2000) & $60-70$ & $65-80$ & $60-80$ & & & & & & $\begin{array}{c}30- \\
40\end{array}$ & \\
\hline & Valencia (2002) & 72 & 83 & 74 & & & & & & & \\
\hline & $\begin{array}{l}\text { Lettinga et al } \\
(1983)\end{array}$ & & & $55-78$ & & & & & & & \\
\hline $\begin{array}{l}\text { UASB - laguna } \\
\text { facultativa }\end{array}$ & CDMB (2006) & 84 & 88 & & & & & & & & \\
\hline $\begin{array}{l}\text { UASB - lodo } \\
\text { activado }\end{array}$ & $\begin{array}{l}\text { Van Haandel - } \\
\text { Lettinga }\end{array}$ & 85-95 & $85-95$ & & & & & $15-25$ & & 10-20 & $70-95$ \\
\hline $\begin{array}{l}\text { UASB - lodo } \\
\text { activado SBR }\end{array}$ & Torres (2000) & $84-86$ & $87-93$ & & & & & $20-90$ & & $\begin{array}{c}23- \\
72 \\
\end{array}$ & \\
\hline $\begin{array}{l}\text { Reactor anaero- } \\
\text { bio de flujo pistón } \\
\text { RAP }\end{array}$ & RAS (2000) & $60-70$ & $65-80$ & $60-80$ & & & & & & $\begin{array}{c}30- \\
40\end{array}$ & \\
\hline $\begin{array}{l}\text { Reactor anaero- } \\
\text { bio de contacto }\end{array}$ & $\begin{array}{l}\text { Rodríguez et al. } \\
\text { (2006) }\end{array}$ & & & $75-90$ & & & & & & & \\
\hline $\begin{array}{l}\text { Reactor anae- } \\
\text { robio de lecho } \\
\text { fluidizado }\end{array}$ & $\begin{array}{l}\text { Rodríguez et al. } \\
\text { (2006) }\end{array}$ & & & $80-85$ & & & & & & & \\
\hline \multirow{4}{*}{$\begin{array}{l}\text { Lodo activado } \\
\text { convencional }\end{array}$} & RAS (2000) & $80-90$ & $80-95$ & $80-95$ & & & $15-20$ & & & $10-25$ & \\
\hline & Yáñez (1995) & $85-98$ & $70-98$ & & & & & & & & $95-98$ \\
\hline & Fair (1954) & $55-95$ & $55-95$ & $50-80$ & & & & & & & $90-98$ \\
\hline & $\begin{array}{l}\text { Von Sperling } \\
\text { (1996) }\end{array}$ & $80-90$ & $85-93$ & & & & & $30-40$ & & $30-45$ & $60-90$ \\
\hline $\begin{array}{l}\text { Lodo activado - } \\
\text { SBR }\end{array}$ & $\begin{array}{l}\text { Von Sperling } \\
\text { (1996) }\end{array}$ & $80-90$ & 85-95 & & & & & $30-40$ & & $30-45$ & $60-90$ \\
\hline
\end{tabular}




\begin{tabular}{|c|c|c|c|c|c|c|c|c|c|c|c|}
\hline \multirow{2}{*}{$\begin{array}{c}\text { TECNOLOGÍA } \\
\text { DE } \\
\text { TRATAMIENTO }\end{array}$} & \multirow[b]{2}{*}{ REFERENCIA } & \multicolumn{10}{|c|}{ EFICIENCIA DE REMOCIÓN (\%) } \\
\hline & & SST & $\mathrm{DBO}_{5}$ & DQO & $\mathrm{N} \mathrm{NH}_{3}$ & N ORG & $\mathrm{N} \mathrm{NO}_{3}$ & $\begin{array}{c}\mathbf{N} \\
\text { TOTAL }\end{array}$ & $\mathrm{P} \mathrm{PO}_{4}$ & $\begin{array}{c}\mathbf{P} \\
\text { TOTAL }\end{array}$ & COLIFORMES \\
\hline $\begin{array}{l}\text { Lodo activa- } \\
\text { do - aireación } \\
\text { prolongada }\end{array}$ & $\begin{array}{l}\text { Von Sperling } \\
\text { (1996) }\end{array}$ & $80-90$ & $93-98$ & & & & & $15-30$ & & $10-20$ & $65-90$ \\
\hline \multirow{3}{*}{$\begin{array}{l}\text { Filtro percolador } \\
\text { alta tasa }\end{array}$} & Yáñez (1995) & $70-90$ & $60-85$ & & & & & & & & $90-95$ \\
\hline & $\begin{array}{l}\text { Metcalf \& Eddy } \\
(2001)\end{array}$ & $60-85$ & $65-80$ & $60-80$ & $8-15$ & & & $15-30$ & & $8-12$ & $90-95$ \\
\hline & $\begin{array}{l}\text { Von Sperling } \\
\text { (1996) }\end{array}$ & $85-95$ & 80-93 & & & & & $30-40$ & & $30-45$ & $60-90$ \\
\hline $\begin{array}{l}\text { Filtro percolador } \\
\text { súper tasa }\end{array}$ & RAS (2000) & $65-85$ & $65-85$ & $65-85$ & $8-15$ & $15-50$ & & & & $8-12$ & \\
\hline Laguna aerobia & Ferrer (2009) & & $60-80$ & & & & & & & & \\
\hline \multirow{3}{*}{ Laguna anaerobia } & Mara (1980) & & $50-85$ & & & & & & & & \\
\hline & Arceivala (1984) & & $30-70$ & & & & & & & & \\
\hline & RAS (2000) & $20-60$ & $50-70$ & & & & & & & & $90-99,99$ \\
\hline \multirow{3}{*}{ Lagunas aireadas } & Mara (1980) & & $80-95$ & & & & & & & & \\
\hline & Mendoza (2000) & & $50-60$ & & & & & & & & \\
\hline & RAS (2000) & $85-95$ & $80-95$ & & & & & & & & $90-99,99$ \\
\hline \multirow{3}{*}{$\begin{array}{l}\text { Lagunas faculta- } \\
\text { tivas }\end{array}$} & RAS (2000) & $63-75$ & $80-90$ & & & & & & & 30 & $90-99,99$ \\
\hline & Fair (1954) & $85-95$ & 90-95 & $70-80$ & & & & & & & $95-98$ \\
\hline & Ýáñez (1995) & 90-99 & $75-95$ & & & & & & & & $98-99,99$ \\
\hline \multirow{2}{*}{$\begin{array}{l}\text { Lagunas madura- } \\
\text { ción }\end{array}$} & Mara (1980) & & 80-95 & & & & & & & & \\
\hline & RAS (2000) & 85-95 & $60-80$ & & & & & & & & $90-99,99$ \\
\hline $\begin{array}{l}\text { Laguna anaerobia } \\
\text { - humedal }\end{array}$ & Caicedo (2005) & 87-93 & $80-90$ & & & & & $37-48$ & & $\begin{array}{c}45- \\
50\end{array}$ & \\
\hline \multirow{3}{*}{ Biodiscos } & $\begin{array}{l}\text { Liu \& Liptack } \\
(2000)\end{array}$ & & $85-95$ & & & & & & & & \\
\hline & $\begin{array}{l}\text { Torres et al. } \\
\text { (2006) }\end{array}$ & 85-95 & 85-93 & & & & & $30-40$ & & $\begin{array}{c}30- \\
45\end{array}$ & $60-90$ \\
\hline & $\begin{array}{l}\text { Metcalf \& Eddy } \\
(2001)\end{array}$ & 80-85 & $80-85$ & $80-85$ & 8-15 & & & $15-20$ & & $10-25$ & \\
\hline
\end{tabular}

Fuente: elaboración propia con base en los autores citados.

\section{Indicadores por tecnologías}

Los indicadores consumo de energía eléctrica, costo de inversión, costo de operación y mantenimiento, área posible construida, producción de lodos y producción de biogás de cada una de las tecnologías se obtuvieron de varios estudios publicados (Crites, 2000; Crittenden, 2005; Droste, 1997; Eckenfelder, 2000; Fresenius, 1991; Lawrence, 1970; Lettinga, 1991; Mara, 1997; Metcalf \& Eddy, 2003; Vieira, 1992; Malina, 1992; Van Haandel, 1994; McCarty, 1985; Arthur, 1983; Arceivala, 1986; Von Sperling, 1996; Rich, 1973; Gloyna, 1971; Barbará, 1981; Fields, 1987; Azevedo, 1976; Ramalho,
1983; EPA, 2003; McGarry, 1970; WEF, 1998). Se utilizaron tales indicadores como parte de lo los parámetros de entrada de la metodología de selección de tecnologías para PTARM.

\section{Calidad requerida en el agua residual tratada (vertimiento)}

Cada país tiene normas de vertimiento de las aguas residuales tratadas para ser descargadas en cuerpos de aguas receptores. En la tabla 3 se observan los criterios de calidad requeridos para el agua residual tratada en varias ciudades y países. 


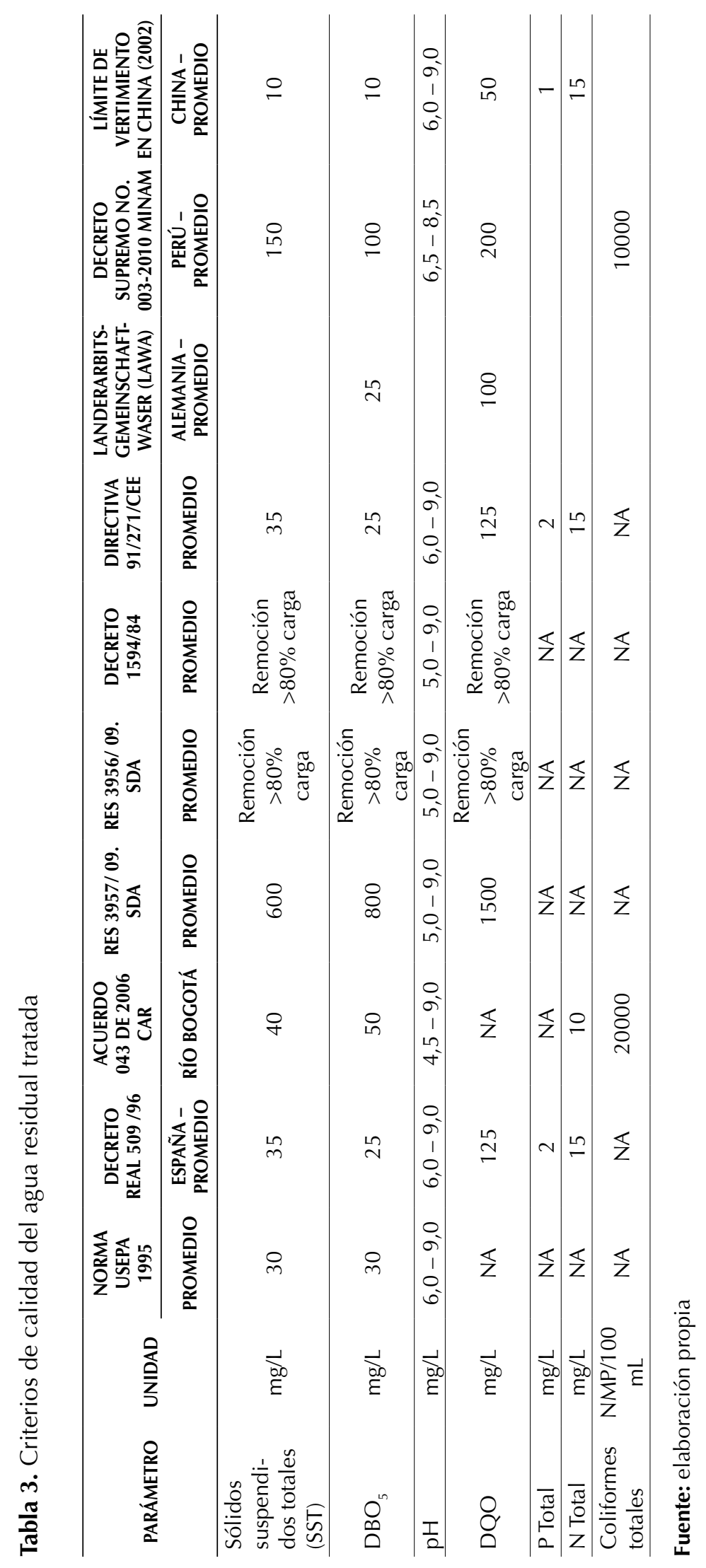




\section{Aspectos de la localización de PTARM}

Las PTARM deben estar localizadas en sitios alejados de ecosistemas críticos y de zonas de recreación. De igual forma, deben estar en un área libre de amenaza por deslizamientos, inestabilidad geológica e inundaciones; además debe proveerse de algún tipo de obra de protección en su perímetro (RAS, 2000; Rodríguez, 2009; Arceivala, 1986; Droste, 1997). La ubicación posible será en el sitio o área donde los vientos regularmente no se desplacen en dirección a la población beneficiada. De manera general, puede ser a más de un kilómetro de distancia de la población (urbanización con viviendas existentes) o puede ser inferior, por ejemplo a 200 $\mathrm{m}$ de distancia mínima a la residencia más próxima a la PTARM, a menos que el estudio de impacto ambiental demuestre la presencia de efectos indeseables para la comunidad cercana (RAS, 2000). La descarga de las aguas residuales tratadas se deberá realizar a un cuerpo de agua receptor de flujo permanente o por infiltración al suelo o terreno.

\section{Metodología de selección de tecnologías}

Para la selección de una PTARM se pueden considerar tres atributos (cualidades o propiedades) significativos de evaluación, que incluyen elementos propios de la idea y perfil de proyecto de PTARM, así como la operación y el mantenimiento de la misma a saber: el atributo técnico, el económico y el ambiental.

Para la cualidad o atributo técnico, considerado como los aspectos propios de la PTARM establecidos con el propósito de cumplir con la normatividad ambiental de vertimiento en cuerpos de aguas receptores y su rendimiento y/o eficiencia para la remoción de contaminantes presentes en el agua residual cruda, se establecen parámetros como: la confiabilidad (operación de la PTARM en condiciones ambientales ajustadas a su sitio de localización, ingreso de carga orgánica adecuada, poca variación en la temperatura (Bernal, 2003) y de concentración de contaminantes presentes en el agua residual); viabilidad (el proceso de construcción debe ser simple y efectuarse en corto plazo); flexibilidad (la tecnología en el tratamiento de aguas residuales debe producir agua residual tratada óptima en forma continua, con un mínimo de operación y de fácil mantenimiento); complejidad (operación con procesos y reactores sencillos y eficaces para tener un rendimiento óptimo en el tratamiento; generalmente los reactores más complejos no resultan siempre los más eficientes); accesibilidad (el grado de acceso a la PTARM debe ser fácil y adecuado con la tecnología seleccionada); requerimiento de análisis y controles (monitoreo constante de variables que afecten o que sean susceptibles en el proceso de tratamiento), tratamiento y manejo de subproductos (biogás y lodos).

La cualidad o atributo económico es considerado como los aspectos relacionados con los costos de inversión, operación y mantenimiento que se puedan generar a partir de la puesta en marcha de la PTARM. Se suelen describir parámetros como: costos por $\mathrm{m}^{3}$ de agua residual tratada (costo de inversión, operación y mantenimiento de la PTARM, es decir la tecnología debe garantizar un agua residual tratada óptima al menor costo posible (Rojas, 2002), requerimiento de área (espacio físico para la instalar la PTARM y necesidades posteriores de expansión), empleo de energía (para la operación de procesos y operaciones unitarias en el tratamiento del agua residual), relación con otros proyectos (la tecnología seleccionada debe tener relación con otros proyectos locales y regionales, con el propósito de lograr la articulación entre proyectos), requerimiento personal calificado (personal con capacidad técnica y alto nivel tecnológico para la operación, mantenimiento y reparación de los procesos y operaciones unitarias de tratamiento de aguas residuales) y participación local (aprovechamiento de oportunidades técnicas, administrativas y legales en el ámbito local de desarrollo del proyecto).

Para la cualidad o atributo ambiental, considerado como las propiedades ambientales que pueda tener la PTARM en términos de los impactos generados en su entorno ,se describen parámetros como: generación de olores, ruido, espumas, 
impacto estético, reutilización del agua residual tratada y estabilidad de la PTARM.

La metodología propuesta de selección de tecnologías de tratamiento de aguas residuales municipales expone una función ponderada de agregación de producto o de promedio geométrico ponderado de la siguiente forma:

$$
I T O_{P T A R M}=\prod_{i=n}^{n} P_{i}^{a_{i}}
$$

Donde $I T O_{\text {PTARM }}$ es el índice de tecnología óptima para las condiciones de evaluación del tratamiento de agua residual municipal por cada tecnología evaluada; $P_{i}$ son los parámetros considerados por cada atributo (técnico, económico o ambiental) y su puntaje es el obtenido por el criterio a evaluar por cada tecnología; y $a_{i}$ es el peso de importancia de cada parámetro según la agregación de producto por cada atributo seleccionado. Para establecer el puntaje por criterio de cada parámetro $\left(P_{i}\right)$ en condiciones desfavorables o favorables, se consideran los siguientes criterios de evaluación:

Tabla 4. Descriptores y cualificación por parámetro

\begin{tabular}{lcl}
\hline DESCRIPTOR & $\begin{array}{c}\text { VALOR } \\
\text { NUMÉRICO } \\
\text { ASIGNADO } \\
(\mathbf{P})\end{array}$ & CUALIFICACIÓN \\
\hline Bajo & 10 & $\begin{array}{l}\text { Importancia relativa más } \\
\text { baja }\end{array}$ \\
\hline Medio & 20 & Importancia relativa media \\
\hline Alto & 30 & Importancia relativa alta \\
\hline
\end{tabular}

Fuente: elaboración propia.

Tabla 5. Matriz de evaluación por tecnología de tratamiento de aguas residuales

\begin{tabular}{|c|c|c|c|c|}
\hline ATRIBUTO & PARÁMETRO & $\begin{array}{c}\text { VALOR } \\
\text { ASIGNADO } \\
\text { (P) }\end{array}$ & $\begin{array}{l}\text { PESO DE } \\
\text { IMPORTANCIA } \\
\text { (a) }\end{array}$ & $\mathbf{p a}$ \\
\hline \multirow{7}{*}{ Técnico (42\%) } & Confiabilidad & & 0,06 & \\
\hline & Viabilidad & & 0,06 & \\
\hline & Flexibilidad & & 0,06 & \\
\hline & Complejidad & & 0,06 & \\
\hline & Accesibilidad & & 0,06 & \\
\hline & Requerimientos de análisis y controles & & 0,06 & \\
\hline & Tratamiento y manejo de subproductos & & 0,06 & \\
\hline \multirow{6}{*}{$\begin{array}{l}\text { Económico } \\
(36 \%)\end{array}$} & Costos (inversión, operación y mantenimiento) & & 0,06 & \\
\hline & Requerimiento de área & & 0,06 & \\
\hline & Empleo de energía eléctrica & & 0,06 & \\
\hline & Relación con otros proyectos & & 0,06 & \\
\hline & Requerimiento de personal calificado & & 0,06 & \\
\hline & Participación local & & 0,06 & \\
\hline \multirow{4}{*}{$\begin{array}{l}\text { Ambiental } \\
(22 \%)\end{array}$} & Generación de olores, ruidos y espumas & & 0,055 & \\
\hline & Impacto estético & & 0,055 & \\
\hline & Reutilización de aguas tratadas & & 0,055 & \\
\hline & Estabilidad de la PTARM & & 0,055 & \\
\hline
\end{tabular}

Fuente: elaboración propia 
A partir de los parámetros y los pesos de importancia asignados por cada parámetro, se desarroIla una matriz de evaluación por cada alternativa de tratamiento de aguas residuales municipales, que permite ejecutar la valoración técnica, económica y ambiental de aspectos cuantitativos y cualitativos de las diferentes opciones de tecnologías (tabla 5).

Por lo tanto, se debe realizar un análisis detallado por cada tecnología a evaluar, para tener una ponderación real y ajustada a las condiciones locales donde se quiere realizar el proyecto de PTARM. Al hacer la evaluación por tecnología mediante el índice ITO $\mathrm{ITARM}_{\text {en }}$ en ponderación global, la tecnología que tenga la menor calificación será la seleccionada como la tecnología óptima de tratamiento de aguas residuales municipales.

\section{Estrategia Espacial para la localización de PTARM}

La evaluación de las PTARM no solo se limita a la evaluación de aspectos cualitativos y cuantitativos, como se mencionó anteriormente, sino que está directamente relacionada con un factor de espacialidad, que involucra tanto las regiones o municipios aledaños a su localización como la población afectada, los sitios de vertimientos a cuerpos de aguas receptores, el espacio físico requerido para su implantación (área disponible), así como con el impacto ambiental negativo causado por la mala disposición de los residuos. Cabe señalar que las aguas residuales son generadas a partir de actividades de tipo doméstico, industrial, comercial, institucional, agrícola y municipal, y la disposición de estas aguas residuales en cuerpos de aguas receptores implican una ubicación espacial (Benujah, 2013).

Dadas la cantidad y complejidad de las variables a evaluar para la adecuada disposición de las PTARM, se hace necesario estructurar la información de forma digital; es decir, vincular procedimientos que permiten la captura, almacenamiento, manipulación, análisis, simulación y salida gráfica de datos geográficamente referenciados (Moreno, 2008; Kang-Tsung, 2012). De esta manera, los elementos constitutivos de la evaluación de las PTARM deben representar un objeto de la superficie terrestre, ya sea espacial o no espacial (límites municipales, afluentes, cabeceras municipales, entre otros), y la representación según su localización geográfica, su forma geométrica, sus relaciones topológicas o grado de conectividad, adyacencia e inclusión que comparte con otros elementos espaciales, además de almacenar otras características no espaciales que los hace disímiles unos de otros (Benujah, 2013; Zhang, 2002), como el caudal, DBO $_{5}$, SST y la densidad poblacional.

Se recomienda entonces la estructuración de una base de datos alfanumérica en un modelo de datos relacional, compuesto básicamente por una serie de tablas, en donde las columnas correspondan a los campos o atributos y las filas hagan referencia a los registros de cada uno de los objetos descritos en las tablas (Silberschatz, 2010). Las tablas o entidades deben almacenar información del mismo tipo en el caso municipal: las características de las tecnologías de tratamiento, eficiencia de remoción y los parámetros para establecer los criterios de descarga de los cuerpos de agua; también se debe establecer una relación con las demás entidades porque comparten algún campo (competencia) entre ellas (Moreno, 2008). Puede utilizarse el lenguaje de consulta SQL tanto para crear tablas como para insertar, borrar o modificar los datos. Los resultados de las consultas que se generan en estas bases de datos son datos particulares o tablas en las que se han establecido condiciones mediante una serie de valores numéricos (Silberschatz, 2010; Worboys, 2004).

Como un estudio de caso específico, se menciona la cuenca del río Bogotá, en el cual la valoración espacial relacionada con las PTARM obedece a las grandes descargas de aguas residuales generadas por los municipios aledaños y que parten desde su nacimiento en Villapinzón hasta la desembocadura en el río Magdalena (en 
los municipios de Ricaurte y Girardot), sin dejar de lado los demás municipios que efectúan la descarga de sus contaminantes a través de algunos drenajes tributarios al río. En la figura 1 se muestran los municipios que cuentan o están en proceso de construcción de una PTARM (color beige); sin embargo, también son plenamente identificables los municipios que no han focalizado sus esfuerzos en el diseño y construcción de PTRAM; la figura muestra además las vías principales y la red de afluentes que desembocan en el río Bogotá.

La metodología del análisis espacial de las variables involucradas para el estudio de las PTARM involucra la preparación de los layers o capas de información digital que se capturan en forma vectorial, ya sea a nivel de punto, líneas o polígonos, y que está directamente relacionada con la estructura de la información recopilada (Fischer, 2006; Smith, 2007). Se hace necesario entonces capturar los límites de los municipios, los afluentes hídricos, la ubicación de las cabeceras municipales, si es posible los puntos de vertimientos, las vías de mayor jerarquía de las zonas aledañas y los datos atributivos vinculados a cada una de las capas mencionadas (Youssef, 2009).

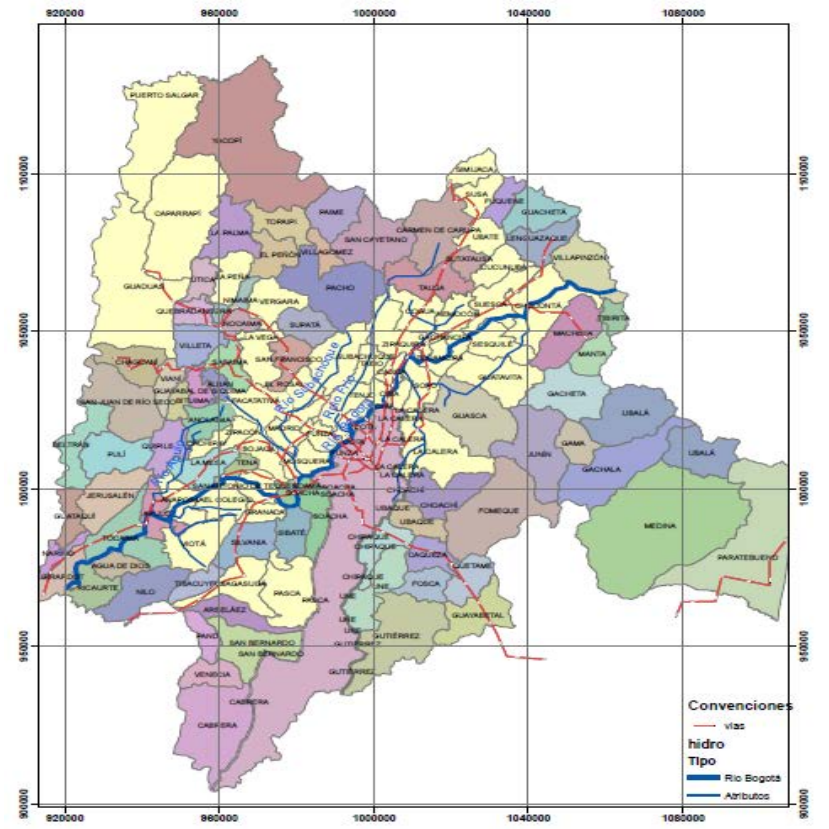

Figura 1. Municipios con PTARM

Fuente: elaboración propia.

En la figura 2 se muestran algunas consultas simples, que relacionan la densidad poblacional con el caudal, la cantidad de $\mathrm{DBO}_{5}$ y SST respectivamente, identificando con color azul los

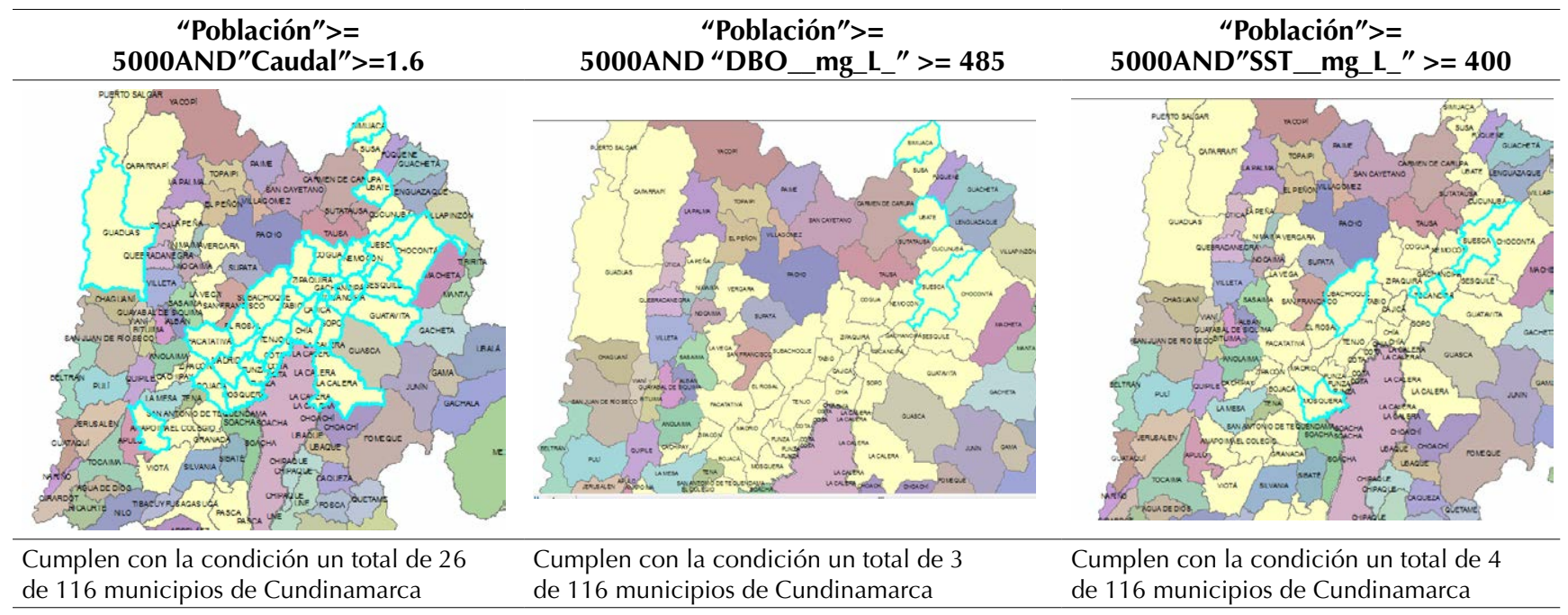

Figura 2. Consultas atributivas simples

Fuente: elaboración propia. 
municipios que cumplen las condiciones relacionadas en cada muestra espacial.

Se infiere entonces que de los municipios con mayor cantidad de habitantes y que cuentan con una PTARM o están en proceso de construcción, $22,4 \%$ presenta un caudal superior a $1,6 \% ; 2,5 \%$ presenta un DBO superior a $485 \mathrm{mg} / \mathrm{L}$ y $3,4 \%$ presenta un SST superior a $400 \mathrm{mg} / \mathrm{L}$.

Una ventaja especial que proporciona la estructuración digital de la información es el análisis geoestadístico; la mayoría de software especializados en los sistemas de información geográfica (SIG), como el ArcGis, Quamtun Gis o el GvSig, presentan módulos para el análisis exploratorio de los datos espaciales y herramientas para crear superficies estadísticas ( (Smith, 2007; Ansaa, 2012). En la figura 3 se presenta una distribución de frecuencia del DBO mediante una serie de barras que agrupan los valores observados de DBO y SST, respectivamente, para los municipios con PTARM.

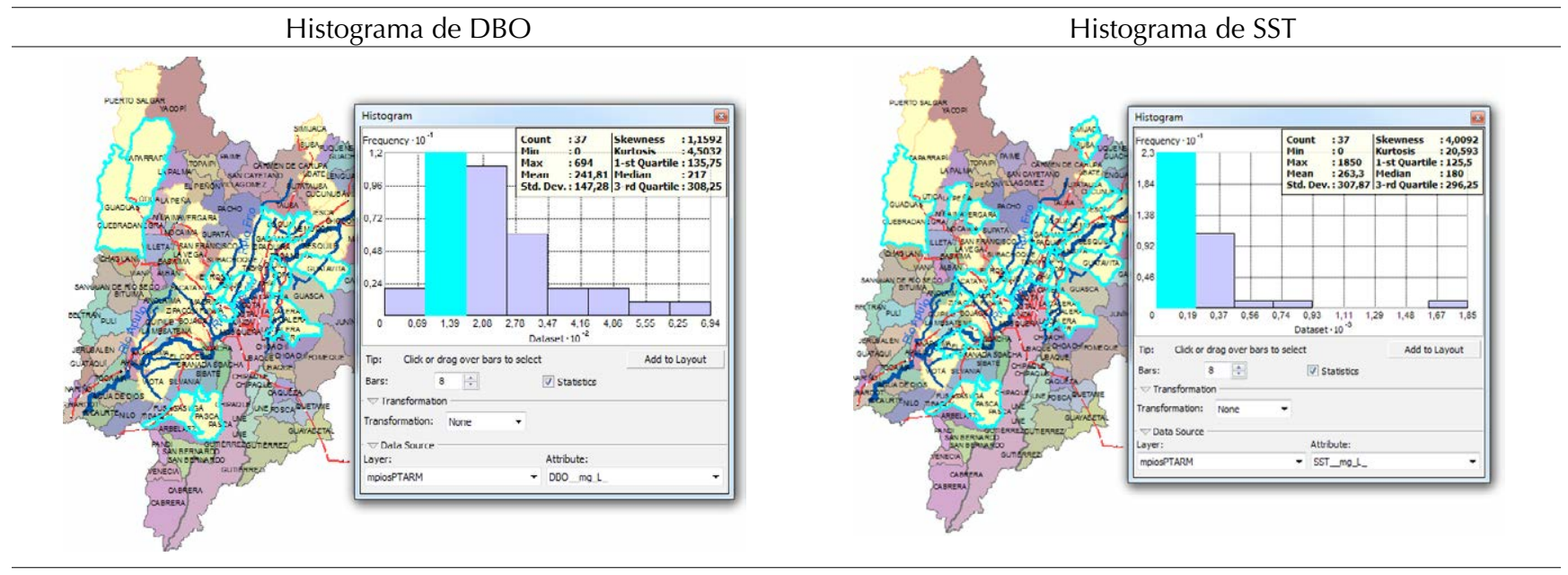

Figura 3. Histograma de valores para el DBO y SST

Fuente: elaboración propia

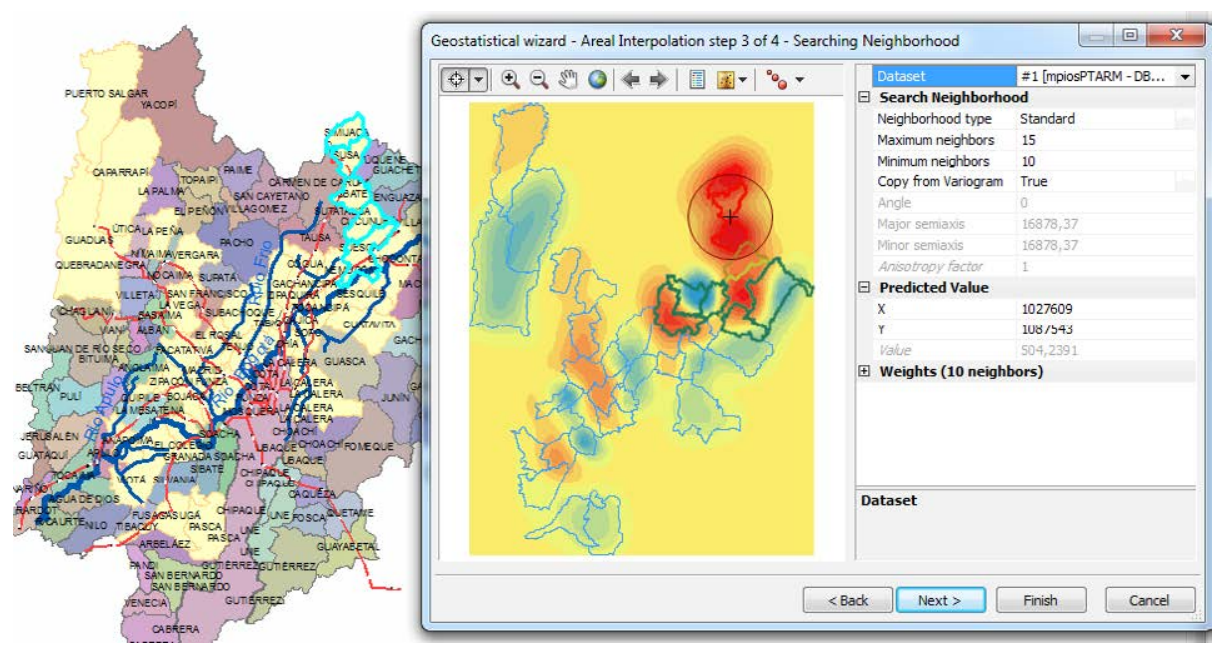

Figura 4. Análisis de autocorrelación espacial

Fuente: elaboración propia. 
Las medidas de centralidad que se aprecian en la figura 3 muestran la distribución de los datos, así como el promedio de la DBO con 241,81 y la del SST en 263,3 que genera el centro de distribución; la mediana representa la proporción acumulada para el DBO en 217 y para el SST de 180, y una desviación estándar de 14,28 y 307,87, respectivamente. Se observa además que los dos histogramas son asimétricamente positivos, es decir que la masa de los valores es inferior a la media. La potencialidad de los datos estructurados consiste en que están estrictamente ligados a la ubicación espacial de manera que se pueden seleccionar los cuartiles como en el ejemplo de mayor frecuencia e identificar al mismo tiempo los municipios que se encuentran en este rango de valores.

Se pueden además construir semivariogramas para determinar la autocorrelación espacial. Los valores de la semivarianza de la figura 4, que corresponde a una imagen raster, permite observar que la autocorrelación de la densidad poblacional es distinta en una y otra dirección; los colores rojo y naranja representan los valores más altos de semivarianza como en el caso del municipio de Simijaca, Suesca, Susa y Ubaté.

El análisis espacial que se puede generar en la evaluación de las PTARM depende en gran medida de la cantidad y calidad de los parámetros ambientales, técnicos y sociales que se obtengan, ya sea a partir de información tomada in situ o a partir de información obtenida mediante dispositivos tecnológicos.

\section{CONCLUSIONES}

La selección de PTARM en países en vía de desarrollo, debe considerar aspectos de la composición típica del agua residual cruda, eficiencia de remoción de contaminantes por tecnología, indicadores de desempeño por tecnología, aspectos ambientales de la localización y la estrategia espacial para la localización de la PTRAM, adecuada en el marco de una planificación ambiental hídrica en la cuenca hidrográfica, para la descontaminación del cuerpo de agua y el establecimiento de una dimensión ambiental pertinente en la protección, manejo y ordenamiento de la cuenca hídrica.

La metodología propuesta expone elementos y aspectos técnicos de construcción multidisciplinaria para la elegibilidad de tecnologías apropiadas al contexto local y regional, que generen confiabilidad, viabilidad, flexibilidad, accesibilidad, controles y análisis de los procesos, así como el tratamiento y manejo de subproductos, que cumplan la normatividad ambiental de descargas al cuerpo de agua o por infiltración al suelo.

De manera específica la ubicación y localización de la PTRAM, debe cumplir con elementos especiales para la óptima selección del sitio posible de ubicación, dado que racionaliza, la utilización de recursos naturales, el empleo de energía y minimiza los posibles efectos ambientales (olores, ruido, espumas, etc.) en la zona de estudio o de intervención en la implantación de las PTARM. Desde luego, el costo de inversión es una variable influente, pero que es proporcionada mediante recursos económicos de la nación mediante políticas y planes específicos de inversión, pero los costos de operación y mantenimiento, los asume el operador y beneficiario del servicio, por ello, lo delicado de la selección de la tecnología de PTARM a utilizar. De acuerdo con lo anterior, debe proporcionarse un marco racional para la evaluación y selección de alternativas de PTARM, que sea de fácil comprensión de los conceptos, técnicas y tecnologías, que conduzcan a una selección estratégica de PTARM y que ayuden a la implementación de tecnologías que alimenten la generación de un desarrollo sostenible en países en vía de desarrollo.

Por otra parte, el costo de inversión es una variable influyente, pero puede ser suplida mediante recursos económicos de la nación mediante políticas y planes específicos de inversión. En cambio, los costos de operación y mantenimiento los asume el operador y beneficiario del servicio, por eso es importante hacer una selección precisa de las tecnologías de PTARM según las necesidades reales de tratamiento. 
De acuerdo con lo anterior, debe proporcionarse un marco racional para la evaluación y selección de alternativas de PTARM que sea de fácil apropiación de conceptos, técnicas y tecnologías, y que conduzcan a una selección estratégica de PTARM y que ayuden a la implementación de tecnologías que alimenten la generación de un desarrollo sostenible en países en vía de desarrollo.

\section{AGRADECIMIENTOS}

Los autores agradecen al Doctorado en Ingeniería, de la Facultad de Ingeniería de la Universidad Distrital Francisco José de Caldas, por el apoyo en la elaboración de este documento. Además, expresan sus agradecimientos al profesor Vidal Fernando Peñaranda Galvis, de la Facultad del Medio Ambiente y Recursos Naturales de la Universidad Distrital Francisco José de Caldas, por la revisión de forma del documento.

\section{REFERENCIAS}

Ansaa, P. (2012). Site Suitability Analysis for a Central Wastewater Treatment Plant(S) In Accra Metropolitan Area Using Geographic Information System.

APHA. (1989). Standard methods for the examination of water and wastewater. Washington: AWWA-WEF.

APHA. (2009). Handbook of Water and Wastewater Treatment Plant Operations, Second Edition. USA: American Public Health Association.

Arceivala D.J. (1986). Wastewater Treatment for Pollution Control. Nueva Delhi: McGraw-Hill.

Arceivala D.J,. (1981). Wastewater Treatment and Disposal: Engineering and Ecology in Pollution control. New York: Marcel Dekker Inc.

Arceivala SJ. (2007). Wastewater treatment for pollution control and reuse. New delhi: Mc Graw Hill.

Arthur J.P. (1983). Notes on the Design and Operation of Waste Stabilization Ponds in Warm Climates of Developing Countries. Washintong: The World Bank.

Azevedo J.M. (1976). Manual de Hidráulica. Mexico: Editorial Harla S. A.
Barbará. (1981). Equipamientos para tratamiento de aguas. Sao Paulo.

Bdour A. (2009). Perspectives on sustainable wastewater treatment technologies and reuse options in the urban areas of the Mediterranean region. Desalination, 162-174.

Benujah, B. (2013). Site Suitability Evaluation For Sewage Treatment Plant In Nagercoil Municipality, Tamil Nadu Using Remote Sensing Techniques.

Bernal D.P. (2003). Guía de selección de tecnologías para el tratamiento de aguas residuales domésticas por métodos naturales. Seminario internacional de métodos naturales para el tratamiento de aguas residuales, (pp. 19-27). Cartagena Colombia.

Bottero M. (2011). Application of the Analytic Hierarchy Process and the Analytic Network Process for the assessment of different wastewater treatment systems. Environmental Modelling \& Software, 1211-1224.

Chung J. (2013). Assessment and selection of best available technology (BAT) for wastewater facilities in the leather tanning and finishing industry. Resources, Conservation and Recycling, 32-37.

Crites R. (2000). Small and descentralized wastewater management systems. New York: Mc Graw Hill.

Crittenden J. (2005). Water treatment: principles and design. Second edition. USA: Wiley \& sons INC.

Droste R. (1997). Theory and practice of water and wastewater treatment. New york: John Wiley \& Sons Inc.

Eckenfelder W. (2000). Industrial water pollution control. New york: Mc Graw Hill.

EPA. (2003). Design Criteria; Rotating Biological Contactors. Ohio USA: EPA.

Fair G. (1968). Abastecimiento de aguas y remoción de aguas residuales. Mexico: Limusa.

Ferrer J. (2008). Tratamiento biológicos de aguas residuales. Valencia: Universidad politécnica de valencia.

Fields J. (1987). Aguas residuales de café. Curso-Seminario de Tratamiento Anaerobio de residuos, (pp. 1-5). Cali.

Fischer, M. (2006). Spatial Analysis and Geocomputation Select essays. 
Fresenius W. (1991). Manual de disposición de aguas residuales. Lima: Centro panamericano de ingeniería sanitaria y ciencias ambientales (CEPIS/OPS/OMS).

Garrido M. (2013). Including the environmental criteria when selecting a wastewater treatment plant. Environmental Modelling \& Software, 1-9.

Geldermann J. (2004). The reference installation approach for the techno-economic assessment of emission abatement options and the determination of BAT according to the IPPC-directive. Journal of Cleaner Production, 389-402.

Gernaey K.V. (2004). Activated sludge wastewater treatment plant modelling and simulation: state of the art. Environmental Modelling \& Software, 763-783.

Gloyna E.F. (1971). Waste Stabilization Ponds. Ginegra: World Health Organization.

Hammer M.J. (2012). Water and wastewater technology. USA: Prentice Hall.

Hernández A. (1996). Depuración de aguas residuales. Madrid España: Servicio de publicaciones de escuela de ingenieros de caminos, canales y puertos.

Hernández F. (2009). Technical efficiency and cost analysis in wastewater treatment processes: A DEA approach. Desalination, 230-234.

Hong Y.S. (2003). Evolutionary self-organising modeIling of a municipal wastewater treatment plant. Water Research, 1199-1212.

Hurtado J. (2000). Metodología de la investigación holística. . Caracas: Fundación SYPAL.

Kalbar P. (2012). Technology assessment for wastewater treatment using multiple-attribute decision-making. Technology in Society, 295-302.

Kang-Tsung, C. (2012). Introduction to Geographic Information Systems.

Lawrence A.W. (1970). Unifield basis for biological treatment. Design and operation. . USA: ASCE.

Lettinga G. (1991). UASB - process design for various types of wastewaters. Water Science and Technology, 87-107.

Malina J.F. (1992). Design of anaerobic processes for the treatment of industrial and municipal waste. Pensilvania USA: Ed. Technomic Publishing Company.

Mara D. (1997). Design manua for waste stabilization ponds in india. . Leeds: University of Leeds.
McCarty P. (1985). Historical Trends in the Anaerobic Treatment of Dilute Wastewaters. Proceedings of the Seminar/Workshop Anaerobic Treatment of Sewage (pp. 3-15). USA: University of Massachusetts at Amherst.

McGarry. M.G. (1970). Stabilization pond design criteria for tropical Asia. Kansas: Ed. R.E. Mckinney, Lawrenc.

Metcalf \& Eddy. (2003). Wastewater engineering: treatment, disposal and reuse. 4 edition. New york: Mc Graw Hill.

Molinos M. (2010). Economic feasibility study for wastewater treatment: A cost-benefit analysis. Science of the Total Environment, 4396-4402.

Moreno, A. (2008). Sistemas y Análisis de la Información Geográfica.

Pophali G. (2011). Optimal selection of full scale tannery effluent treatment alternative using integrated AHP and GRA approach. Expert Systems with Applications, 10889-10895.

Ramalho R. (1983). Tratamiento de aguas residuales. Quevec: Editorial Reverte S.A.

RAS. (2000). Reglamento técnico de agua potable y saneamiento básico. RAS 2000. Titulo E. Tratamiento de Aguas residuales. Bogotá: Ministerio de Desarrollo Económico.

Rich L.G. (1973). Environmental systems engineering. USA: Mc Graw Hill.

Rodriguez G. (2011). Environmental and economic profile of six typologies of wastewater treatment plants. water research, 5997-6010.

Rodríguez J.P. (2012). Assessment of an aerobic treatment system for. Int. J. Environmental Engineering, 307-314.

Rodríguez, J. P. (2009). Selección técnico económico del sistema de depuración de aguas residuales. Aplicando la evaluación de la descontaminación hídrica. Tecnología del agua, 22-21.

Rojas R. (2002). Sistemas de Tratamiento de Aguas Residuales. "GESTIÓN INTEGRAL DE TRATAMIENTO DE AGUAS RESIDUALES" (pp. 8-15). LIMA: OPS.

Romero J. (2004). Tratamiento de aguas residuales. Teoría y principios de diseño. . Bogotá: Escuela Colombiana de Ingeniería. 
Sala-Garrido R. (2011). Comparing the efficiency of wastewater treatment technologies through a DEA metafrontier model. Chemical Engineering Journal, 766-772.

Schoenberger H. (2009). Integrated pollution prevention and control in large industrial installations on the basis of best available techniques - The Sevilla Process. Journal of Cleaner Production, 1526-1529.

Silberschatz, A. (2010). Database System Concepts.

Smith, M. (2007). Geospatial Analysis. 71.

Urkiaga A. (2006). Methodologies for feasibility studies related to wastewater reclamation and reuse projects. Desalination, 263-269.

Van Haandel A.C. (1994). Anaerobic Sewage Treatment. A practical guide for regions with a hot climate. Inglaterra: John Whiley and sons.

Vergel G. (2010). Metodología. Un manual para la elaboración de diseños y proyectos de investigación. Compilación y ampliación temática. Barranquilla: Publicaciones Corporación UNICOSTA.

Vieira S.M. (1992). Sewage treatment by UASB reactor. Operation results and recommendations for design and utilization. Wat. Sci. Tech.,, 143-157.

Vieira, S. (1986). Development of technology for the use of the UASB reactor in domestic sewage treatment. Wat. Sci. Tech, 143-157.
Villaseñor J. (2001). Eliminación biológica de fósforo en aguas residuales. Castilla: Universidad de Castilla.

Von Sperling M. (1996). Comparison among the most frequently used systems for wastewater treatment in developing countries. Water science and technology., 156-180.

WEF. (1998). Design of Municipal Wastewater Treatment Plants. Manual of Practice $n^{\circ} 8$, New York: Am. Soc. Civ.Eng.

Worboys, M. (2004). Gis a Computing Perspective Second Edition .

Yañez F. (1995). Lagunas de estabilización. Lima: Centro panamericano de ingeniería sanitaria y ambiental.

Youssef, A. (2009). Geotechnical investigation of sewag wastewater disposal sites and use of GIS land use maps to assess environment hazards.

Zeng G. (2007). Optimization of wastewater treatment alternative selection by hierarchy grey relational analysis. Journal of Environmental Management, 250-259.

Zhang, J. \&. (2002). Uncertainty in Geography Information.

\section{(C) $(1) \Theta$}

Tecnura • p-ISSN: 0123-921X • e-ISSN: 2248-7638• Vol. 19 No. $46 \bullet$ Octubre - Diciembre $2015 \bullet$ pp. 149-164 